\begin{tabular}{|c|c|c|c|c|c|c|c|c|}
\hline Species & Location & $\begin{array}{l}\text { Month of } \\
\text { collection }\end{array}$ & Habitat & $\begin{array}{l}\text { Avg length; } \\
\text { Diameter (cm) }\end{array}$ & $\begin{array}{l}\text { Total segments / } \\
\text { Ciltellar segments / } \\
\text { Type of clitellum }\end{array}$ & $\begin{array}{l}\text { Prosto- } \\
\text { mium }\end{array}$ & $\begin{array}{l}\text { Location of } \\
\text { prostate } \\
\text { glands }\end{array}$ & $\begin{array}{l}\text { Spermathecae } \\
\text { number / } \\
\text { Location }\end{array}$ \\
\hline $\begin{array}{l}\text { Lennogaster } \\
\text { chittagongensis } \\
\text { Stephenson, } 1917\end{array}$ & $\begin{array}{l}\text { Nathwai, } \\
\text { Sukhna WLS }\end{array}$ & $\begin{array}{l}\text { September, } \\
2002\end{array}$ & Soil rich in moisture & $2.4-7 ; 0.15$ & 120; XIII-XVII; A & Epi & $\mathrm{XVII}$ & $1 ; 8$ \\
\hline $\begin{array}{l}\text { Lennogaster pusillus } \\
\text { Stephenson, } 1920\end{array}$ & $\begin{array}{l}\text { Kansal forest, } \\
\text { Chandigarh (U.T. }\end{array}$ & August, 2000 & $\begin{array}{l}\text { Soils rich in organic } \\
\text { matter, leaf litter and }\end{array}$ & $\begin{array}{l}2.5-4.5 ; 0.13 \\
\text { humus }\end{array}$ & 100-125; XIII-XVII; A & Pro-epi & $\mathrm{XVII}$ & $1 ; 8$ \\
\hline $\begin{array}{l}\text { Octochaetona beatrix } \\
\text { Beddard, } 1902\end{array}$ & Hoshiarpur & March, 2001 & $\begin{array}{l}\text { Earth of potted } \\
\text { plants }\end{array}$ & $7-8 ; 0.35$ & $\begin{array}{l}\text { 170-180; XIII-XVII, } \\
\text { XVIII; A }\end{array}$ & Epi & XVII \& XIX & $2 ; 8,9$ \\
\hline $\begin{array}{l}\text { Ramiella } \\
\text { bishamberensis } \\
\text { Stephenson, } 1914\end{array}$ & $\begin{array}{l}\text { Forest near } \\
\text { Sukhna lake, } \\
\text { Chandigarh (U.T. }\end{array}$ & $\begin{array}{l}\text { August, } 2000 \\
\text { Г.) }\end{array}$ & Moist neutral soils & $3.5 ; 0.1$ & 85; XIV-XVI; A & Epi & XVII-XIX & $2 ; 8,9$ \\
\hline $\begin{array}{l}\text { Family: Moniligastri } \\
\text { Drawida japonica } \\
\text { Michaelson, } 1917\end{array}$ & $\begin{array}{l}\text { dae } \\
\text { Solan }\end{array}$ & June, 2003 & $\begin{array}{l}\text { Soils rich in organic } \\
\text { matter }\end{array}$ & $3-6 ; 0.26$ & $\begin{array}{l}\text { 130-142; } \\
\text { inconspicuous clitellum }\end{array}$ & Pro & $x$ & $1 ; 7 / 8$ \\
\hline
\end{tabular}

Tany - Tanylobous; Pro - Prolobous; Epi - Epilobous; S - Saddle; A - Annular

NOTE

ZOOS' PRINT JOURNAL 21(1): 2137-2139

\section{ICHTHYOFAUNA FROM FOUR DISTRICTS OF MARATHWADA REGION, MAHARASHTRA, INDIA}

\section{C.J. Hiware}

Reader, Department of Zoology, Dr. Bababsaheb Ambedkar Marathwada University, Aurangabad, Maharashtra 431004, India Email: drhiware@rediffmail.com

The Marathwada region of Maharashtra is one of the richest in aquatic resources that includes tributaries of river Godavari, Purna, Painganga, Manjara and Dudhana apart from reservoirs and lakes. In the field of ichthyology valuable contributions have been made by Ahirrao and Mane (2000) who studied ichthyofauna from Parbhani district of Maharashtra state and Sakhare (2001) who studied ichthyofauna of Jawalgaon Reservior in Solapur district in Maharashtra state. The present work was mainly undertaken to investigate the fish diversity from this region and it is first effort in this direction.

The survey work was mainly undertaken in four major districts, namely, Aurangabad, Nanded, Parbhani and Osmanabad of Marathwada region. From these districts some of the places having small and big water resources with fishery potential were selected for collection of ichthyofauna. Some of the important places under study include:

\section{Aurangabad district:}

Collection sites: Aurangabad, Paithan, Werul, Ajantha, Sillod. Water resources: Godavari river, Kham river, Nathsagar dam, Purna river, Salim Ali lake, Harsool lake, Tisgaon pond, Khajan pond, etc.

\section{Parbhani district:}

Collection sites: Parbhani, Yeldari, Purna, Jintur etc.

Water resources: Purna river, Kapra river, Masoli lakes, Yeldari dam, Jam pond, Rahati pond, etc.

\section{Nanded district:}

Collection sites: Nanded, Vishnupuri, Kandhar, Kinwat, Mahur Water resources: Godavari river, Manar river, Painganga river, Vishnupuri dam, Jagatunga Samudra reservoir, Dhamdari pond.

\section{Osmanabad district:}

Collection sites: Osmanabad, Tuljapur, Naldurg, Kallam, Bhum Water resources: Terna river, Manjara river, Sina river, Bori dam, Tugao pond, etc.

Fishes were collected during regular monthly between May 2003 and April 2004 from the local fish markets and fishing spots of different places. The local fishermen fish using different types of gill nets, cast nets, Maccharjali, disco nets operated through indigenously designed rafts and Ratnagiri type boats. After systematic identification the specimens were preserved in $4 \%$ formalin and deposited in the Zoology Department Museum, Dr. Babasaheb Ambedkar Marathwada University Aurangabad after providing appropriate registration numbers and other details. The identification of the fishes were carried out with the help of standard literature (Day, 1878; Jayram, 1981; Qureshi \& Qureshi, 1983; Datta \& Srivastava, 1988; Talwar \& Jhingran, 1991).

Sixty-six fish species belonging to 33 genera, 16 families and eight orders were recorded from the freshwaters of four districts of Marathwada region in Maharashtra state (Table 1). The ichthyofauna consisted of carps, catfishes and trash fishes. The carps dominated overall over other groups throughout the year. The group also supported capture as well as culture fishery

Manuscript 1210: Received 28 May 2004: Revised received 16 July 2004: Finally accepted 20 September 2005 ; Date e organisation; www.zoosprint.org 
Table 1. Ichthofauna collected from selected sites of four districts of Marathwada region

\begin{tabular}{|c|c|c|c|c|c|}
\hline $\begin{array}{l}\text { Species } \\
\text { Class: Pisces }\end{array}$ & $\begin{array}{l}\text { Registration } \\
\text { Number }\end{array}$ & $\begin{array}{l}\text { Date of } \\
\text { collection }\end{array}$ & \multicolumn{2}{|c|}{ Locality with districts } & Habitat \\
\hline $\begin{array}{l}\text { Class: Pisces } \\
\text { Subclass: Teleostei } \\
\text { Order: Clupeiformes } \\
\text { Suborder: Notopteroidei } \\
\text { Family: Notopteridae }\end{array}$ & & & & & \\
\hline Notopterus notopterus (Pallas) & 30 & $4 / 11 / 03$ & Aurangabad & Aurangabad & Lake \\
\hline Notopterus chitala (Ham.) & 29 & $4 / 11 / 03$ & Aurangabad & Aurangabad & Lake \\
\hline \multicolumn{6}{|l|}{$\begin{array}{l}\text { Order: Cypriniformes } \\
\text { Suborder: Cyprinoidei } \\
\text { Family: Cyprinidae }\end{array}$} \\
\hline Chela argentea (Cuv. \& Val.) & 43 & $8 / 1 / 04$ & Nanded & Nanded & River \\
\hline Chela phulo (Ham.) & 53 & $5 / 2 / 04$ & Daultabad & Aurangabad & Pond \\
\hline Chela sladoni (Day.) & 54 & $15 / 02 / 2004$ & Naldurg & Osmanabad & Reservoir \\
\hline Cyprinus corpio. (Linn.) & 07 & $22 / 06 / 2003$ & Daultabad & Aurangabad & Pond \\
\hline Catla catla (Ham.) & 01 & $28 / 05 / 2003$ & Paithan & Aurangabad & Reservoi \\
\hline Cirrhinus mrigala (Ham.) & 55 & $25 / 02 / 2004$ & Paithan & Aurangabad & Reservoir \\
\hline Cirrhinus reba (Ham.) & 12 & $13 / 07 / 2003$ & Kinwat & Nanded & River \\
\hline Cirrhinus flungee (Ham.) & 63 & $6 / 4 / 04$ & Kinwat & Nanded & River \\
\hline Ambylpharyngodon microlepis (Bleeker) & 52 & $3 / 2 / 04$ & Paithan & Aurangabad & Reservoir \\
\hline Ambylpharyngodon mola (Ham.) & 20 & $13 / 09 / 2003$ & Vishnupuri & Nanded & Reservoir \\
\hline Discognathus modestus (Hacker) & 57 & $21 / 03 / 2004$ & Parbhani & Parbhani & Pond \\
\hline Discognathus lamta (Ham.) & 34 & $28 / 11 / 2003$ & Werul & Aurangabad & Lake \\
\hline Labeo rohita (Ham.) & 08 & $22 / 06 / 2003$ & Daultabad & Aurangabad & Pond \\
\hline Labeo calbasu (Ham.) & 59 & $25 / 03 / 2004$ & Paithan & Aurangabad & Reservoir \\
\hline Labeo boggut (Skyes) & 64 & $6 / 4 / 04$ & Kinwat & Nanded & River \\
\hline Labeo fimbriatus (Bloch) & 40 & $29 / 12 / 2003$ & Vishnupuri & Nanded & Reservoir \\
\hline Osteobrama cotio (Ham.) & 32 & $24 / 11 / 2003$ & Paithan & Aurangabad & Reservoir \\
\hline Osteobrama belkeri (Skyes) & 31 & $14 / 11 / 2003$ & Nanded & Nanded & River \\
\hline Puntius amphibias (Valeneiennes) & 36 & $5 / 12 / 03$ & Aurangabad & Aurangabad & Pond \\
\hline Puntius chola (Ham.) & 47 & $3 / 2 / 04$ & Naldurg & Osmanabad & Reservoir \\
\hline Puntius conchonius (Ham.) & 48 & $3 / 2 / 04$ & Naldurg & Osmanabad & Reservoir \\
\hline Puntius jerdoni (Day) & 66 & $19 / 04 / 2004$ & Parbhani & Parbhani & Pond \\
\hline Puntius sarana sarana (Ham.) & 49 & $19 / 04 / 2004$ & Parbhani & Parbhani & Pond \\
\hline Puntius ticto ticto (Ham.) & 37 & $5 / 12 / 03$ & Aurangabad & Aurangabad & Pond \\
\hline Puntius sophore. (Ham.) & 50 & $3 / 2 / 04$ & Tuljapur & Osmanabad & Reservoir \\
\hline Puntius stigma (Day) & 13 & $15 / 07 / 2003$ & Tuljapur & Osmanabad & Reservoir \\
\hline Hypothalamichthys molitrex (Val.) & 22 & $13 / 09 / 2003$ & Vishnupuri & Nanded & Reservoir \\
\hline Thynnichthys sandkhol (Skyes) & 51 & $3 / 2 / 04$ & Paithan & Aurangabad & Reservoir \\
\hline Tor tor (Ham.) & 23 & $21 / 09 / 2003$ & Kinwat & Nanded & River \\
\hline Barilius barila (Ham.) & 09 & $12 / 7 / 03$ & Naldurg & Osmanabad & Reservoir \\
\hline Barilius bendelisis (Ham.) & 11 & $12 / 7 / 03$ & Naldurg & Osmanabad & Reservoir \\
\hline Barilius barna (Ham.) & 10 & $12 / 7 / 03$ & Naldurg & Osmanabad & Reservoir \\
\hline Rasbora daniconius (Ham.) & 38 & $6 / 12 / 03$ & Daultabad & Aurangabad & Pond \\
\hline Ctenopharyngodon idella (Ham.) & 42 & $8 / 1 / 04$ & Nanded & Nanded & River \\
\hline \multicolumn{6}{|l|}{ Family: Cobitidae } \\
\hline Lepidocephalichthys guntea (Ham.) & 39 & $6 / 12 / 03$ & Daultabad & Aurangabad & Pond \\
\hline Nemacheilus aureus (Ham.) & 41 & $29 / 12 / 2003$ & Vishnupuri & Nanded & Reservoir \\
\hline Nemacheilus beavani (Ham.) & 46 & $21 / 01 / 2004$ & Purna & Parbhani & River \\
\hline Nemacheilus botia (Ham.) & 35 & $29 / 11 / 2003$ & Aurangabad & Aurangabad & Pond \\
\hline \multicolumn{6}{|l|}{$\begin{array}{l}\text { Order: Siluriformes } \\
\text { Family: Bagridae }\end{array}$} \\
\hline Mystus aor. (Ham.) & 24 & $2 / 10 / 03$ & Paithan & Aurangabad & Reservoir \\
\hline Mystus bleekeri (Day) & 25 & $7 / 10 / 03$ & Purna & Parbhani & River \\
\hline Mystus cavasius (Ham.) & 18 & $1 / 9 / 03$ & Werul & Aurangabad & Lake \\
\hline Mystus seenghala (Sykes) & 19 & $1 / 9 / 03$ & Werul & Aurangabad & Lake \\
\hline Mystus tengara (Dum) & 60 & $28 / 03 / 2004$ & Jintur & Parbhani & Pond \\
\hline Mystus vittatus (Bloch) & 44 & $12 / 1 / 04$ & Parbhani & Parbhani & Pond \\
\hline Rita rita (Ham.) & 45 & $12 / 1 / 04$ & Parbhani & Parbhani & River \\
\hline \multicolumn{6}{|l|}{ Family: Claridae } \\
\hline Clarias batrachus (Linnaeus) & 05 & $15 / 06 / 2003$ & Aurangabad & Aurangabad & Lake \\
\hline Clarias gariepinus (Cuv-val) & 06 & $18 / 06 / 2003$ & Mahur & Nanded & River \\
\hline \multicolumn{6}{|l|}{ Family: Heteropneustidae } \\
\hline \multicolumn{6}{|l|}{ Family: Schilbeidae } \\
\hline Pseudeudeutropius taakree (Bleeker) & 65 & $17 / 04 / 2004$ & Purna & Parbhani & River \\
\hline \multicolumn{6}{|l|}{ Family: Siluridae } \\
\hline Wallago attu (Bloch and Schneider) & 17 & $7 / 8 / 03$ & Paithan & Aurangabad & Reservoir \\
\hline Ompak bimaculatus (Bloch) & 16 & $7 / 8 / 03$ & Paithan & Aurangabad & Reservoir \\
\hline
\end{tabular}




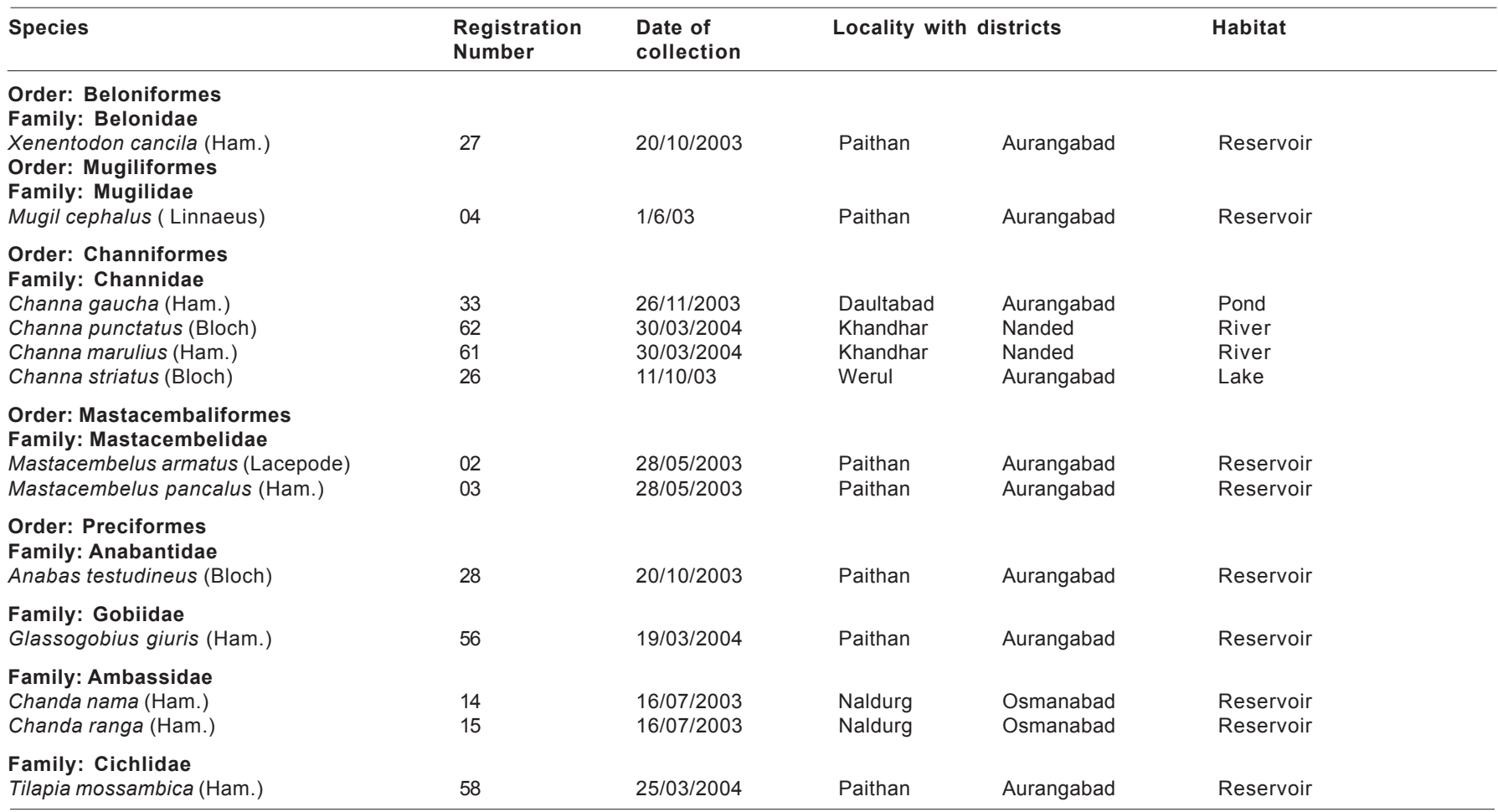

of the region. The catfishes are important for capture fishery.

The species of Channa, Mastacembelus, Clarias, Heteropnestus, Anabus etc. have air breathing organs and fetch good market value as live fish. Puntius sophore, $P$. sarana sarana, P. ticto ticto, P. stigma, P. amphibias, P. jerdoni, Nemacheilus botia, Lepidocephalichthys guntea, Chanda nama, C. ranga, Ambylpharyngodon microlepis have ornamental value due to small size and bright colours and can are used as aquarium fishes.

\section{REFERENCES}

Ahirroa, S.D. and A.S. Mane (2000). The diversity of ichthyofauna, taxonomy and fisheries from some fresh waters of Parbhani district (MS). Journal of Aquatic Biology 15(1\&2) : 40-43.

Datta, M. and M.P. Shrivastava (1988). Natural History of Fishes and Systematic of Freshwater Fishes of India. Narendra Publishing House, Delhi.

Day, F.S. (1978). The Fishes of India, William and Sons Ltd., London.

Jayaram, K.C. (1981). The Freshwater Fishes of India. A Handbook Zoological Survey of India, Calcutta.

Jhingran, V.G. (1975). Fish and Fisheries of India. Hindustan Publishing Corporation, India.

Qureshi, T.A. and N.A. Qureshi (1983). Indian Fishes. BRIJ Brothers Sultana Road, Bhopal.

Sakhare, V.B. (2001). Ichthyofauna of Jawalgaon reservoir in Solapur district (MS). Journal of Aquatic Biology 16(1\&2): 31-33.

Talwar, P.K. and A.G. Jhingran (1991). Inland Fishes of India and Adjacent Countries. Vol. I \& II. Oxford \& IBH Publ. Co. Pvt. Ltd, New Delhi.

\section{ACKnOWledgement}

Authors are thankful to UGC, New Delhi for providing the financial support through major research project sanctioned vide Ref. No.F-3130/2003 (SR) on 30 March 2003 and to Head and Professor., Department of Zoology for providing necessary laboratory and library facilities.

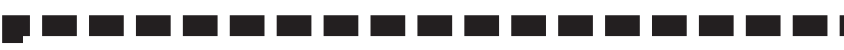

Threatened Taxa Monitoring System for south asian fauna, flora and fungi

launched!

Visit

\section{www. southasiantaxa.org}

for more information.

The website will be put up in three phases. The first part of First Phase is uploaded with most current taxonomic and conservation information on selected groups of fauna and flora.

If you are interested in helping with this website, please write to us at

\section{TTMS}

c/o ZOO, WILD, CBSG South Asia 29-1 Bharathi Colony

Peelamedu, Coimbatore

Tamil Nadu 641004, India

zoocrew@vsnl.net, enquiry@southasiantaxa.org 